diagnosis of psoriasis. $12.3 \%$ presented with active polyarthritis at the initial diagnosis of PsA. There was a significant difference in the use of systemic therapy in females, in which there was a higher rate of systemic therapy used in female PsA patients prior to developing PsA as compared to females with psoriasis who did not develop PsA ( $n=24,48 \%$ vs $n=16,16 \% ; p<0.001)$. There was no significant association between ethnicity, education level, comorbidities, BMI, body area affected and family history of psoriasis with development of PsA. The predictive factors in developing PsA are females $(\mathrm{OR}=3.14,95 \% \mathrm{Cl} 1.77,5.58)$, presence of nail involvement $(\mathrm{OR}=3.72,95 \% \mathrm{Cl} 1.91,7.26)$ and the use of systemic therapy (OR $=3.04,95 \% \mathrm{Cl} 1.70,5.43)$, (all $p$ values $<0.001$ ).

Conclusion: This study highlighted that female sex, presence of nail involvement and use of systemic therapy prior to PsA diagnosis are predictive risk factors in developing PsA among patients with underlying psoriasis. Further prospective studies with larger cohorts are needed to better delineate these risk factors. REFERENCES:

[1] Mohd Affandi A, Khan I, Ngah Saaya N. Epidemiology and Clinical Features of Adult Patients with Psoriasis in Malaysia: 10-Year Review from the Malaysian Psoriasis Registry (2007-2016). Dermatology research and practice. 2018;2018:4371471.

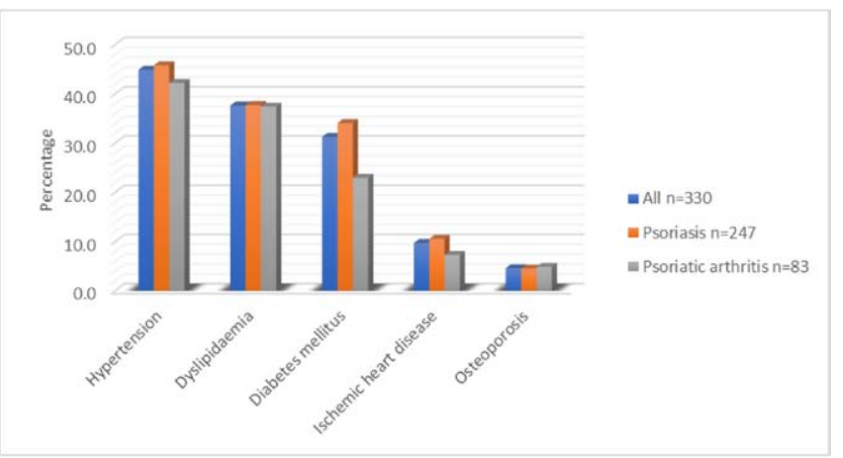

Figure 1. Comorbidities among Patients with underlying Psoriasis and Psoriatic Arthritis $(n=330)$

Chi-square test revealed that there was no significant difference between psoriasis and psoriatic arthritis $(p>0.05)$

Disclosure of Interests: WAI YANG LOO: None declared, FARIZ YAHYA Speakers bureau: speaker for Novartis, Gilead, AbbVie, Janssen, Eli Lilly, ZueIlig-Pharma and Pfizer., Consultant of: consultancy work with Novartis, Gilead, AbbVie, Eli Lilly, Zuellig-Pharma and Pfizer., Grant/research support from: research grants from Novartis, Gilead, AbbVie, Boehringer-Ingelheim and Pfizer., WINN HUI HAN: None declared, NIK AIMEE AZIZAH FAHEM: None declared, SHIN SHEN YONG: None declared, Lydia Say Lee Pok: None declared, Zhenli Kwan Speakers bureau: Novartis, Zuellig, YING CHEW TEE: None declared. DOI: 10.1136/annrheumdis-2021-eular.2792

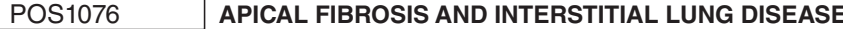 IN PATIENTS WITH PSORIATIC ARTHRITIS: DO WE} UNDERESTIMATE?

G. Ayan ${ }^{1}$, E. B. Ata ${ }^{2}$, G. Durhan ${ }^{3}$, M. Ariyurek ${ }^{3}$, U. Kalyoncu ${ }^{1} .{ }^{1}$ Hacettepe University Medical Faculty, Department of Internal Medicine Division of Rheumatology, Ankara, Turkey; ${ }^{2}$ Hacettepe University Medical Faculty, Department of Internal Medicine, ANKARA, Turkey; ${ }^{3}$ Hacettepe University Medical Faculty, Department of Radiology, ANKARA, Turkey

Background: Extra-articular manifestation (EAMs) definition is not clearly defined in psoriatic arthritis (PsA). Nail involvement, enthesitis, dactylitis has been widely studied however, data are needed on pulmonary involvement in PsA. Objectives: We aimed to understand real-life results of lung involvement in PsA patients.

Methods: From the PsA cohort followed in our outpatient clinic, patients who have been requested a chest computed tomography (CT) for any reason by any department were retrieved from medical records and included in this retrospective cross-sectional analysis. All CTs were assessed by a radiologist who is blinded to the patients ' clinical history and findings were categorized as parenchymal, airway, pleural findings and lymphadenopathy (LAM). Moreover, any findings that are radiologically attributed to a specific entity such as previous tuberculosis $(\mathrm{Tb})$ infection were noted. Demographic/clinic data including smoking status, concomitant lung problems, disease characteristics (duration, axial/ peripheral involvement) were collected and analyzed.

Results: A total of $80 / 1072(7.4 \% ; 65 \%$ female) PsA patients with mean (SD) age 56.1 (13.2) years were included in the study. Median (IQR) PsA duration was 23.5 (55.75) months and 36 (45\%) patients had peripheral, 29 (36.3\%) patients had axial involvement. For the rest of $15(18.7 \%)$ patients, radiographic
Table 1. Patient characteristics with apical fibrosis, interstitial lung disease

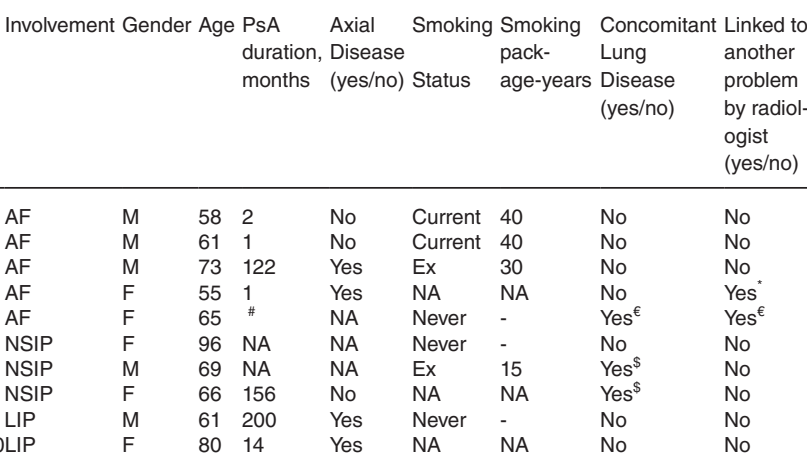

$\mathrm{AF}=$ Apical fibrosis; NSIP= Non-spesific interstitial pneumonia, LIP= Lymphocytic interstitia pneumonia; PsA= Psoriatic Arthritis, NA= Not available*radiotherapy sequelae; \# tomography was done prior to PsA diagnosis; $€$ previous tuberculosis infection; $\$$ chronic obstructive pulmonary disease

assessment was missing. Smoking status could be retrieved in 47 patients (never $=40.4 \%$, ex-smoker $=19.1 \%$, current smoker $=40.4 \%$ ). There were 14 concomitant lung problem in $12(15 \%)$ patients. CT findings showed that $68.8 \%$ of patients had at least one lung pathology. Parenchymal findings were seen in $65 \%$ of the patients as both non-spesific changes (atelectasis, $n=35$; nodules, $\mathrm{n}=24$; ground glass opacity, $\mathrm{n}=9$; sequelae fibrosis, $\mathrm{n}=9$; emphysema, $\mathrm{n}=7$; consolidation, $n=5$, interstitial thickening, $n=5$; pulmonary cyst, $n=4$ ) and specific pathologies as apical fibrosis (AF) $(n=5)$ and intersititial lung disease (ILD) $(n=5$, $\mathrm{NSIP}=3, \mathrm{LIP}=2$ ). AF was linked to previous $\mathrm{Tb}$ infection in 1 patient and radiotherapy in another (Table 1). However, there is no specific pathology in the rest of $3(3.7 \%)$ patients that AF could be attributed to and all of them were male. Other pathologies were seen as airway findings in $28.8 \%$ patients (bronchiectasis, $n=$ 17 , bronchial wall thickness, $n=10$, air trapping $n=7$, centrilobular opacity, $n=4$, and pleural findings in $13.8 \%$ of the patients (pleural plaque, $n=5$, effusion, $n=$ 3 , thickness, $n=3$ ). LAM was observed in around $4 \%$ of the patients.

Conclusion: The real-life experience of our PsA cohort showed that pulmonary findings included variable spesific, non-spesific findings. As a limitation results represent the CT findings of around $8 \%$ of our PsA cohort. Within the scope of previous reports male predominant AF and ILD come forward in PsA that requires further attention in future studies $[1,2]$.

REFERENCES:

[1] Peluso R, lervolino S, Vitiello M, et al (2015) Extra-articular manifestations in psoriatic arthritis patients. Clin Rheumatol 34 (4):745-753.

[2] Bargagli E, Bellisai F, Mazzei MA, et al (2020) Interstitial lung disease associated with psoriatic arthritis: a new disease entity? Intern Emerg Med.

Disclosure of Interests: None declared.

DOI: 10.1136/annrheumdis-2021-eular.2873

\section{POS1077 CLINICAL AND RADIOLOGICAL FEATURES OF SEROPOSITIVE PSORIATIC ARTHRITIS}

H. EL Moutaouakil ${ }^{1}$, K. Nassar ${ }^{1}$, S. Janani ${ }^{1}{ }^{1}$ University Hospital of Ibn Rochd, Department of Rheumatology, casablanca, Morocco

Background: Rheumatoid factor (RF) and anti-cyclic citrullinated peptides (anti-CCP) are two highly specific laboratory markers for rheumatoid arthritis However, they can also be found in other chronic inflammatory arthritis such as psoriatic arthritis (Psa)

Objectives: The aim of our study is to analyze the different epidemiological and paraclinical characteristics of seropositive psoriatic arthritis.

Methods: Descriptive and analytical retrospective study from January 2010 to December 2020 conducted in the Department of Rheumatology of the University Hospital of Ibn Rochd, Casablanca.

Inclusion criteria: patients diagnosed with psoriatic arthritis according to the CASPAR or ASAS 2009 criteria, regardless of the immunological status (RF/ anti-CCP).

The patients were then divided into two groups: seropositive (positive anti-CCP and / or positive RF) and seronegative. A univariate analytical study was performed by jamovi version 1.2.27

Results: 80 patients were enrolled. 22 patients were seropositive (Group 1) and 58 were seronegative (Group 2). In the group 1, the mean age was 51.1 years (+/- 9.45). The sex ratio $M / F$ was 0.29 , the mean age of onset was 43.5 years $(+/$ 10.4), the mean duration of disease was $9.55+/-8$ years. All patients had skin involvement. Polyarticular involvement was present in all cases; axial involvement was present in $68.18 \%$ of cases and enthesitis in $68.18 \%$ of cases. All patients were HLA B 27 negative. The presence of X-ray and / or ultrasound bone 
Table 1. Comparison of characteristics of seropositive and seronegative PsA patients

\begin{tabular}{llll}
\hline Items & $\begin{array}{l}\text { Group 1 } \\
\text { Seropositive patients }(\mathrm{n}=22)\end{array}$ & $\begin{array}{l}\text { Group 2 } \\
\text { Seronegative patients } \\
(\mathrm{n}=58)\end{array}$ & p-value \\
\hline Age (years) & $51.1+/-9.45$ & $50.7+/-15.8$ & 0.9 \\
Sex ratio M/F & 0.29 & 1.14 & 0.013 \\
Age onset (years) & $43.5+/-10.4$ & $37.4+/-16.5$ & 0.123 \\
Skin involvement $(\%)$ & 100 & 50 & 0.009 \\
Bone erosions (\%) & 81.1 & 56.9 & $<0.001$ \\
Response to treatment (\%) & 70.58 & 51.14 & $<0.001$ \\
\hline
\end{tabular}

erosions in addition to signs of destruction was in $81.8 \%$ of cases. The response to treatment with (corticosteroids / NSAIDs / DMARDs) was partial in $70.58 \%$. In group 2, the mean age was 50.7 years $(+/-15.8)$. The sex ratio $\mathrm{M} / \mathrm{F}$ was 1.14 , the mean age of onset was 37.4 years $(+/-16.5)$, the mean duration of disease was 13.3 years $(+/-9.5)$. Skin involvement was present in $50 \%$ of patients. Polyarticular involvement was present in all cases, axial involvement was present in $67.24 \%$ of cases and enthesitis in $56.9 \%$ of cases. All patients were HLA B 27 negative. The presence of X-ray and / or ultrasound bone erosions in addition to signs of destruction was in $56.9 \%$ of cases. The response to treatment with (corticosteroids / NSAIDs / DMARDs) was partial in $57.14 \%$

Conclusion: The presence of anti-CCP according to several studies is linked to the presence of deformities, dactylites and radiological erosions $(1,2)$. In our series, seropositivity was accompanied by the erosive nature of the destruction, as well as a tendency to resistance to treatment.

REFERENCES:

[1] Kim KY, Lee YH. Anti-cyclic citrullinated peptide antibody in psoriatic arthritis: a meta-analysis of its frequency and association with clinical features. $Z$ Rheumatol. mai 2020;79(4):397-403.

[2] Hagiwara S, Tsuboi H, Terasaki T, Terasaki M, Toko H, Shimizu M, et al. Association of anti-cyclic citrullinated peptide antibody with clinical features in patients with psoriatic arthritis. Modern Rheumatology. 3 mars 2020;30(2):365-72.

Disclosure of Interests: None declared.

DOI: 10.1136/annrheumdis-2021-eular.2958

\section{POS1078 CURRENT REGISTRIES IN PSA DO NOT WELL REFLECT THE PROFILES OF PATIENTS WITH PSA BECAUSE THEY ORIGINATE IN SIMILAR COUNTRIES: A SYSTEMATIC LITERATURE REVIEW OF 27 REGISTRIES, OR 16183 PATIENTS}

G. S. Moysidou ${ }^{1}$, K. Aouad ${ }^{1}$, A. Rakotozafiarison 1 , B. Fautrel ${ }^{1}$, L. Gossec ${ }^{1}$ ${ }^{1}$ Sorbonne Université - INSERM; AP-HP, Pitié Salpêtrière Hospital, Rheumatology department, Paris, France

Background: Psoriatic arthritis (PsA) is a multidimensional inflammatory disease with a great geographic variability and a global average prevalence estimated at 133 every

100,000 subjects according to a recent systematic review and meta-analysis (1). Registries and cohorts reflect more closely real-world data than randomized controlled trials (RCTS) and may indicate ongoing interest of each country on PsA. Objectives: The purpose of this study is to assess how recent registries and PsA related cohorts reflect its worldwide prevalence.

Methods: A systematic literature review was performed in Pubmed Medline (PROSPERO

CRD42020175745) to identify all articles reporting on either registries or longitudinal cohorts in PsA, published between 2010 and March 2020. Registries centered on drugs or not PsA-specific, trials and long term extension studies were excluded. The data collection comprised registries' and cohorts' originating countries, patient characteristics and the clinical outcome measures reported. Statistics were descriptive.

Results: Of 673 articles, 73 were relevant for analysis, corresponding to 27 registries or PsA specific cohorts, with the participation of 30 countries. The overall number of patients was 16,183 with a mean of 599 per study. Overall, $50.1 \%$ were men, weighted mean age was 50,6 years and weighted mean disease duration was 6.9 years.

Most of the registries were based in Europe (67\%) or North America (26\%) whereas Africa was underrepresented (Figure 1). USA was the most represented country participating in 6 registries. Mean age and mean disease duration were shorter in international registries (Table 1). Caspar diagnostic criteria were the most frequently used, mainly in the national registries $(86,4 \%)$, whereas the use of diagnostic criteria was more heterogenous in the international registries. Conclusion: Recent registries and PsA specific cohorts do not cover the worldwide spectrum of the disease.

\section{REFERENCES:}

[1] Scotti L, Franchi M, Marchesoni A, Corrao G. Prevalence and incidence of psoriatic arthritis: A systematic review and meta-analysis. Semin Arthritis Rheum. 2018 Aug;48(1):28-34

Table 1. Description of 27 ongoing PsA registries or PsA cohorts, comparing nationwide and international registries

\begin{tabular}{lll}
\hline & NATIONWIDE & INTERNATIONAL \\
& REGISTRIES (N=22) & REGISTRIES (N=5) \\
\hline WOMEN (\%) & 49,4 & 50,8 \\
MEAN AGE, WEIGTHED (YEARS) & 52,32 & 48,09 \\
$\begin{array}{c}\text { MEAN DISEASE DURATION, WEIGHTED } \\
\quad 8,59\end{array}$ & 5,86 \\
$\begin{array}{l}\text { (YEARS) } \\
\text { CASPAR DIAGNOSTIC CRITERIA (\%) }\end{array}$ & 86,4 & 40 \\
\hline
\end{tabular}

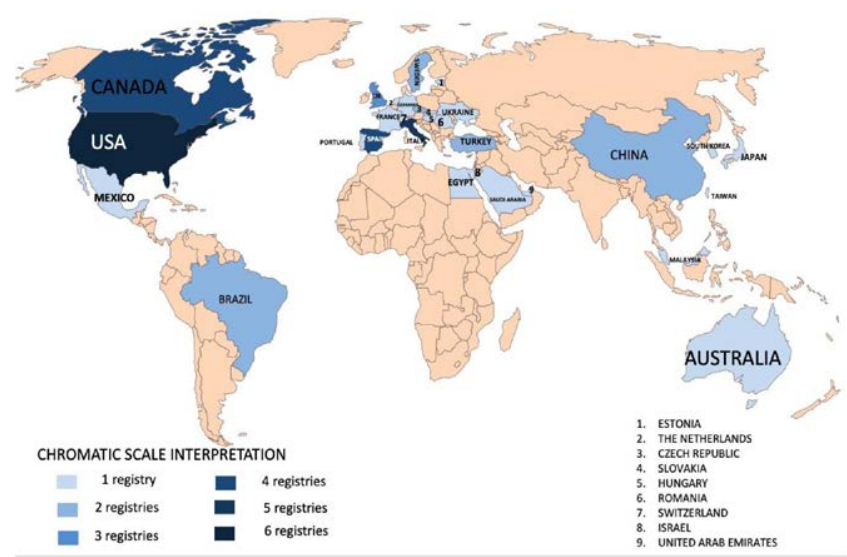

Figure 1. Geographical distribution of PsA registries

Disclosure of Interests: None declared.

DOI: 10.1136/annrheumdis-2021-eular.3097

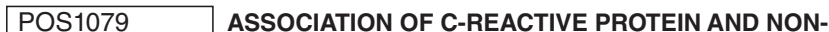 STEROIDAL ANTI-INFLAMMATORY DRUGS WITH CARDIOVASCULAR EVENTS IN PATIENTS WITH PSORIATIC ARTHRITIS: A TIME-DEPENDENT Cox REGRESSION ANALYSIS}

S. H. M. Lam ${ }^{1}$, H. So ${ }^{1}$, I. T. Cheng ${ }^{1}$, E. LI ${ }^{1}$, P. C. Wong ${ }^{1}$, T. K. LI ${ }^{1}$, A. P. W. Lee ${ }^{1}$, L. S. Tam ${ }^{1} .{ }^{1}$ The Chinese University of Hong Kong, Department of Medicine and Therapeutics, Sha Tin, Hong Kong (SAR)

Background: Psoriatic arthritis (PsA) is associated with accelerated atherosclerosis due to underlying inflammation. Whether inflammatory burden and drugs used to suppress inflammation over time are associated with cardiovascular (CV) events remains unclear.

Objectives: This study aims to examine the time-varying effect of C-reactive protein (CRP) levels and the use of drugs including non-steroidal anti-inflammatory drugs (NSAIDs) on the risk of CV events independent of traditional CV risk factors in PsA patients.

Methods: A retrospective cohort analysis was performed in patients with PsA who were recruited from 2008 to 2015 and followed till the end of 2019. The outcome was occurrence of a first CV event. Framingham risk score (FRS) was used to quantify the traditional CV risk. Cox proportional hazard models with time-varying CRP levels and drugs used were analyzed to identify the risk factors for CV events in PsA patients.

Results: 200 patients with PsA (median age: 47.5[40.0 - 56.0]; male: 119 [59.5\%]) were recruited (Table 1. next page). After a mean follow-up of $8.8 \pm 3.8$ years, $30(15 \%)$ patients developed a first CV event. The Kaplan-Meier survival analysis indicated a significant difference in the $\mathrm{CV}$ event-free survival between patients with and without CRP level $>3 \mathrm{mg} / \mathrm{L}$ (Figure $1 \mathrm{~A}$ ) and an inverse relationship between time-varying NSAIDs exposure and CV event-free survival (Fig ure 1B). The multivariable Cox regression model showed that time-varying CRP level (HR 1.02, 95\% Cl 1.00 to 1.04) and NSAIDs exposure (HR 0.30, 95\% Cl 0.15 to 0.95 ) were significantly associated with $\mathrm{CV}$ events after adjusting for baseline FRS (HR 5.04, 95\% Cl 1.83 to 13.85). 